\title{
Originales
}

\section{Estimación pronóstica en enfermos críticos. Validación de un nuevo y muy sencillo sistema de estimación pronóstica de supervivencia en pacientes ingresados en una Unidad de Cuidados Intensivos}

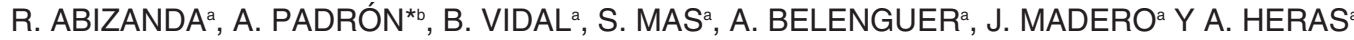 \\ aServei de Medicina Intensiva. Hospital Universitario Asociado General de Castellón. Castellón. España. \\ 'Servicio de Cuidados Intensivos. Hospital Hermanos Amejeiras. La Habana. Cuba.
}

Objetivo. Realizar la validación de un nuevo sistema de estimación pronóstica de supervivencia de enfermos críticos (EPEC) atendidos en una Unidad de Cuidados Intensivos (UCI) polivalente.

Diseño y ámbito. Análisis prospectivo de una cohorte de pacientes atendidos en la UCI de un Servicio de Medicina Intensiva polivalente de un hospital docente de referencia, dotado de $19 \mathrm{ca}-$ mas.

Pacientes y método. Cuatrocientos ochenta y cuatro pacientes ingresados consecutivamente durante 6 meses de 2003. Recogida de datos de un conjunto mínimo básico de datos (CMBD) propio que incluye datos de identificación de pacientes (género, edad, etc.), motivo de ingreso y procedencia del mismo, estimación pronóstica de supervivencia mediante EPEC, MPM II 0 y SAPS II (considerados estos dos como gold stan-

\footnotetext{
*In memoriam.

Correspondencia: Dr. R. Abizanda i Campos.

Servei de Medicina Intensiva.

Hospital Universitario Asociado General de Castellón.

Avda. Benicàssim, s/n.

12004 Castellón. España.

Correo electrónico: abizanda_ric@gva.es rabizandac@terra.es

Manuscrito aceptado el 23-I-2006.
}

dard). La mortalidad fue evaluada al alta hospitalaria.

La validación de EPEC fue realizada mediante análisis de su capacidad discriminante (curvas ROC); la calibración de su capacidad pronóstica (test de Hosmer Lemeshow C) y resolución de las tablas de contingencia $2 \times 2$ alrededor de distintos valores de probabilidad $(20,50,70$ y valor medio de la estimación pronóstica). Se calculó la tasa estandarizada de mortalidad (SMR) para cada uno de los métodos.

Se estableció la regresión lineal de EPEC respecto de MPM II 0 y SAPS II y se realizaron análisis de concordancia (test de Bland-Altman) de la predicción de mortalidad por los tres sistemas.

Resultados. A pesar de una aparentemente buena correlación lineal, una exactitud de predicción y una capacidad de discriminación similares, EPEC no está bien calibrado (ninguna probabilidad de muerte superior al 50\%) y los análisis de concordancia demuestran que más del $10 \%$ de los pares están fuera del intervalo de confianza del $95 \%$.

Conclusión. A pesar de su sencillez de aplicación y cálculo y de incorporar como variable el retraso de ingreso en UCI, EPEC no ofrece ninguna ventaja predictiva sobre MPM II 0 o SAPS II, y sus predicciones se ajustan peor a la realidad.

PALABRAS CLAVE: mortalidad, pronóstico de mortalidad, MPM II O, SAPS II, EPEC, calibración, discriminación, exactitud, tasa estandarizada de mortalidad. 
PROGNOSTIC ESTIMATION IN CRITICAL PATIENTS. VALIDATION OF A NEW AND VERY SIMPLE SYSTEM OF PROGNOSTIC ESTIMATION OF SURVIVAL IN AN INTENSIVE CARE UNIT

Objective. To make the validation of a new system of prognostic estimation of survival in critical patients (EPEC) seen in a multidisciplinar Intensive care unit (ICU).

Design and scope. Prospective analysis of a patient cohort seen in the ICU of a multidisciplinar Intensive Medicine Service of a reference teaching hospital with 19 beds.

Patients and method. Four hundred eighty four patients admitted consecutively over 6 months in 2003. Data collection of a basic minimum data set that includes patient identification data (gender, age), reason for admission and their origin, prognostic estimation of survival by EPEC, MPM II 0 and SAPS II (the latter two considered as gold standard). Mortality was evaluated on hospital discharge.

EPEC validation was done with analysis of its discriminating capacity (ROC curve), calibration of its prognostic capacity (Hosmer Lemeshow C test), resolution of the $2 \times 2$ Contingency tables around different probability values $(20,50,70$ and mean value of prognostic estimation). The standardized mortality rate (SMR) for each one of the methods was calculated.

Linear regression of the EPEC regarding the MPM II 0 and SAPS II was established and concordance analyses were done (Bland-Altman test) of the prediction of mortality by the three systems.

Results. In spite of an apparently good linear correlation, similar accuracy of prediction and discrimination capacity, EPEC is not well-calibrated (no likelihood of death greater than $50 \%$ ) and the concordance analyses show that more than $10 \%$ of the pairs were outside the $95 \%$ confidence interval.

Conclusion. In spite of its ease of application and calculation and of incorporating delay of admission in ICU as a variable, EPEC does not offer any predictive advantage on MPM II 0 or SAPS II, and its predictions adapt to reality worse.

KEY WORDS: mortality, mortality prognosis, MPM II 0, SAPS II, EPEC, calibration, discrimination, accuracy, standardized mortality rate.

\section{INTRODUCCIÓN}

La preocupación por establecer una predicción pronóstica de supervivencia en los enfermos atendidos en los Servicios de Medicina Intensiva es una constante de la investigación clínica en este campo de la asistencia.

A esta inquietud responde el diseño de EPEC (estimación pronóstica en enfermos críticos $)^{1,2}$, descrito en 2002 por un grupo de clínicos cubanos, y cuya finalidad es proporcionar un nuevo instrumento predictivo caracterizado por su sencillez de aplicación y por estar pensado para prescindir de elementos informáticos o de computación matemática que realicen los complejos cálculos que exige una estimación pronóstica. La descripción del formulario de cómputo de probabilidad pronóstica mediante EPEC se presenta en el anexo 1.

De las condiciones teóricas que exige la construcción de un índice pronóstico de supervivencia: sencillez, reproducibilidad y validación en una muestra distinta de la utilizada para su diseño $0^{3,4}$, la única que EPEC no cumple en su descripción inicial ${ }^{1}$ es la de validación en una muestra independiente.

El objetivo de nuestro trabajo tiene tres vertientes: utilizar el sistema EPEC en su aplicación a una cohorte de enfermos críticos, validar su diseño en una muestra independiente y comparar la exactitud de las predicciones obtenidas con la de dos sistemas de estimación pronóstica, ampliamente introducidos en la práctica habitual de las Unidades de Cuidados Intensivos (UCI), y de prestaciones bien conocidas, MPM II 0 y SAPS II; todo ello revisando, al mismo tiempo, la metodología de validación del diseño de un sistema pronóstico de supervivencia.

\section{PACIENTES Y MÉTODOS}

En un Servicio de Medicina Intensiva, de un Hospital docente de referencia con 19 camas (15 de UCI propiamente dichas y 4 de Cuidados Intermedios), y durante un período de 6 meses del año 2003, se ha recogido de forma prospectiva información suficiente para cumplimentar un CMBD (conjunto mínimo básico de datos) de diseño propio.

El CMBD incluía datos demográficos y epidemiológicos (género, edad, motivo de ingreso, procedencia y tipo de paciente), de funcionamiento del Servicio (estancia, gravedad [sistema SAPS II] y carga de trabajo de enfermería [sistema NEMS, con puntuaciones durante el primer día de estancia y del total de todos los días de estancia]), de resultado asistencial (mortalidad hospitalaria) y de estimación pronóstica de la misma (MPM II 0, SAPS II y EPEC).

La capacidad de discriminación pronóstica por los tres sistemas utilizados (MPM II 0, SAPS II y EPEC) fue establecida mediante el cálculo del área bajo la curva ROC (receiver operating characteristics).

La calibración fue establecida mediante el test C de Hosmer Lemeshow y la exactitud de predicción fue calculada mediante resolución de tablas de contingencia de Stuart Kendall, del tipo $2 \times 2$, calculadas para puntos de corte de predicción del $20 \%$, $50 \%$ y $70 \%$, y de las predicciones medias de mortalidad para MPM II 0, SAPS II y EPEC. La resolución de estas tablas permitió el cálculo de la especificidad (E), sensibilidad (S), valor predictivo positivo (VP+), valor predictivo negativo (VP-) y exactitud de predicción. 
ANEXO 1

\section{Descripción de estimación pronóstica en enfermos críticos}

El sistema EPEC, descrito por Padrón et al en 2002¹, constituye un valioso intento de proporcionar un instrumento de estimación pronóstica de supervivencia basado en la simplicidad de su aplicación práctica. Diseñado mediante procedimientos de regresión logística, su principal valor radica en la asunción de que el clínico, a pie de cama, puede no disponer de los medios informáticos precisos para aplicar una ecuación de estimación pronóstica convencional (APACHE, SAPS, etc.) ${ }^{2}$.

Por esta razón los autores intentaron proporcionar al posible usuario una «traducción simple», que mediante una suma y una multiplicación, le permitiera establecer una estimación pronóstica de mortalidad (y por tanto, una estimación inversa de supervivencia). El método queda resumidamente descrito en la siguiente tabla, y los autores enfatizan, en su descripción original, que la capacidad pronóstica queda «refinada» (incrementa su exactitud) si se realizan estimaciones secuenciales, siendo esta exactitud máxima cuando se combinan las predicciones calculadas durante los primeros tres días de estancia en UCI.

\section{Cálculo de estimación pronóstica en enfermos críticos (EPEC)}

\begin{tabular}{|c|c|}
\hline Parámetro & Puntos \\
\hline \multicolumn{2}{|l|}{ Edad (en años) } \\
\hline$<44$ & 2 \\
\hline $45-54$ & 3 \\
\hline $55-64$ & 5 \\
\hline $65-74$ & 7 \\
\hline$>74$ & 11 \\
\hline \multicolumn{2}{|l|}{ Comorbilidades } \\
\hline Fracaso renal crónico & 30 \\
\hline Insuficiencia hepática & 30 \\
\hline Enfermedad pulmonar obstructiva & 15 \\
\hline Diabetes mellitus tipo I & 10 \\
\hline Cardiopatía isquémica & 5 \\
\hline Retraso en el ingreso en UCI & Horas \\
\hline \multicolumn{2}{|l|}{$\mathrm{RCP}$ previa al ingreso en UCI } \\
\hline Sí & 40 \\
\hline No & 0 \\
\hline Frecuencia cardíaca (<60 o > 180 lpm) & 10 \\
\hline Presión arterial media $(<56$ o > 146 mmHg) & 10 \\
\hline Cociente $\mathrm{PO}_{2} / \mathrm{FIO}_{2}(<300 \mathrm{mmHg})$ & 10 \\
\hline Puntuación de la escala de Glasgow & 15 -valor del Glasgow \\
\hline Nivel sérico de potasio $(<2,5 \circ>6,0 \mathrm{mEq} / \mathrm{l})$ & 10 \\
\hline Ventilación mecánica & 35 \\
\hline
\end{tabular}

El resultado numérico de la consideración de los distintos componentes de EPEC facilita un valor que oscila entre 0 y 300, el cual, una vez multiplicado por la constante 0,333 , proporciona la probabilidad de muerte expresada en porcentaje, que oscila entre 0 y 100. RCP: resucitación cardiopulmonar.

La concordancia de las predicciones realizadas por cada uno de los sistemas se estudió mediante el método de análisis de concordancia de Bland Altman, con cálculo de los márgenes de oscilación de los límites del IC 95\% (intervalo de confianza del 95\% de la diferencia media de probabilidad de predicción para cada paciente) y del número de puntos situados fuera del IC 95\%, admitiéndose un 5\%, por definición, como cifra tolerable.

En todos los casos los niveles de significación se establecieron si $\mathrm{p}<0,05$. La abreviatura NS (no significativo) se utilizó cuando p $>0,05$.

El estudio estadístico se realizó mediante el programa SPSS/PC 11.0.

\section{RESULTADOS}

Se han incluido en el estudio 484 pacientes consecutivos no seleccionados, de los cuales fallecieron (mortalidad hospitalaria) 60 pacientes $(12,4 \%)$.

Las tablas 1, 2 y 3 muestran la cifra absoluta de pacientes fallecidos respecto de los predichos muertos para cada intervalo de probabilidad. Las diferencias en el número de pacientes incluidos en cada una de las tablas se justifica por el hecho de no disponer de los datos necesarios para establecer el pronóstico de mortalidad. Las tablas proporcionan, igualmente, los valores de la ratio de la estandarizada (SMR) y su IC $95 \%$.

TABLA 1. Predicciones mediante EPEC, a distintos niveles de probabilidad de fallecimiento

\begin{tabular}{|c|c|c|c|c|}
\hline Probabilidad & Vivos & Muertos & Muertos predichos & Suma \\
\hline $0-\leq 10 \%$ & 275 & 10 & 14,25 & 285 \\
\hline$>10-\leq 20 \%$ & 107 & 15 & 18,3 & 122 \\
\hline$>20-\leq 30 \%$ & 32 & 22 & 13,5 & 54 \\
\hline$>30-\leq 40 \%$ & 9 & 11 & 7 & 20 \\
\hline \multirow[t]{2}{*}{$>40 \%$} & 1 & 1 & 0,8 & 2 \\
\hline & 424 & 59 & 53,85 & 483 \\
\hline
\end{tabular}

IP estimación pronóstica en enfermos críticos (EPEC) promedio 10,46; SMR (tasa estandarizada de mortalidad) = real/predicha = 1,09 (IC 95\%: 0,832-1,41). 
TABLA 2. Predicciones mediante MPM II 0, a distintos niveles de probabilidad de fallecimiento

\begin{tabular}{|l|c|c|c|c|}
\hline \multicolumn{1}{|c|}{ Probabilidad } & Vivos & Muertos & Muertos predichos & Suma \\
\hline $0-\leq 10 \%$ & 184 & 5 & 9,45 & 189 \\
$>10-\leq 20 \%$ & 114 & 6 & 18 & 120 \\
$>20-\leq 30 \%$ & 39 & 4 & 10,75 & 43 \\
$>30-\leq 40 \%$ & 32 & 2 & 11,9 & 34 \\
$>40-\leq 50 \%$ & 10 & 6 & 7,2 & 21 \\
$>50-\leq 60 \%$ & 15 & 6 & 11,55 & 11 \\
$>60-\leq 70 \%$ & 7 & 4 & 7,15 & 18 \\
$>70-\leq 80 \%$ & 10 & 8 & 13.5 & 18 \\
$>80-\leq 90 \%$ & 8 & 10 & 15,3 & 484 \\
\end{tabular}

MPM 0 promedio: 24,54; tasa estandarizada de mortalidad $=$ real/predicha $=0,508($ IC 95\%: 0,388-0,655).

TABLA 3. Predicciones mediante SAPS II, a distintos niveles de probabilidad de fallecimiento

\begin{tabular}{|l|c|c|c|c|}
\hline \multicolumn{1}{|c|}{ Probabilidad } & Vivos & Muertos & Muertos predichos & Suma \\
\hline $0-\leq 10 \%$ & 179 & 4 & 9,15 & 183 \\
$>10-\leq 20 \%$ & 89 & 11 & 15 & 100 \\
$>20-\leq 30 \%$ & 34 & 10 & 11 & 44 \\
$>30-\leq 40 \%$ & 5 & 5 & 3,5 & 10 \\
$>40-\leq 50 \%$ & 17 & 8 & 11,25 & 25 \\
$>50-\leq 60 \%$ & 6 & 0 & 3,3 & 9 \\
$>60-\leq 70 \%$ & 4 & 5 & 5,85 & 20 \\
$>70-\leq 80 \%$ & 11 & 9 & 15 & 1 \\
$>80-\leq 90 \%$ & 0 & 1 & 1 & 399 \\
$>90 \%$ & 1 & 0 & 76,05 & 3 \\
\hline
\end{tabular}

SAPS II promedio: 19,35; tasa estandarizada de mortalidad = real/predicha $=0,697$ (IC 95 \%: 0,522-0,912).

Los análisis de significación de las comparaciones entre la SMR de EPEC y de MPM II 0 y de SAPS II se muestran en la tabla 4, que permite comprobar cómo no existen diferencias significativas entre la SMR de los dos índices más consolidados y sí la hay entre la capacidad de predicción de cualquiera de ellos y EPEC.

La resolución de las tablas de contingencia elaboradas sobre probabilidades de muerte del $20 \%$,

TABLA 4. Significación de las comparaciones de la capacidad gobal de predicción de SAPS II, MPM II 0 y EPEC

\begin{tabular}{|l|c|c|}
\hline Comparación & Ratio SMR (IC 95\%) & $\mathrm{p}$ \\
\hline EPEC frente a MPM 0 & $2,31(1,59-3,37)$ & $<0,05$ \\
EPEC frente a SAPS II & $1,78(1,15-2,51)$ & $<0,05$ \\
MPM 0 frente a SAPS II & $0,736(0,5-1,09)$ & NS \\
\hline
\end{tabular}

No significativo (NS): $\mathrm{p}>0,05$

TABLA 5. Tablas de contingencia $2 \times 2$ y su resolución a distintas probabilidades de predicción de mortalidad mediante EPEC

\begin{tabular}{|lcc|}
\hline Probabilidad & $20 \%$ & $50 \%$ \\
Sensibilidad & 57,62 & 100 \\
Especificidad & 9,25 & 0 \\
Valor predictivo positivo & 8,17 & 12,21 \\
Valor predictivo negativo & 62,68 & 0 \\
Exactitud & 15,73 & 12,21 \\
\hline
\end{tabular}

EPEC: estimación pronóstica en enfermos críticos.
$50 \%$ y $70 \%$ se presenta en las tablas 5,6 y 7 . Debe destacarse que ninguno de los pacientes evaluados mediante EPEC presentó una probabilidad de muerte mayor del $50 \%$, por lo que en este caso la tabla correspondiente muestra únicamente los datos de resolución alrededor de probabilidades del $20 \%$ y $50 \%$.

La tabla 8 ofrece los resultados de la resolución de las tablas de contingencia alrededor tanto de la

TABLA 6. Tablas de contingencia $2 \times 2$ y su resolución a distintas probabilidades de predicción de mortalidad mediante SAPS II

\begin{tabular}{|lccc|}
\hline Probabilidad & $20 \%$ & $50 \%$ & $70 \%$ \\
Sensibilidad & 71,69 & 28,30 & 18,86 \\
Especificidad & 22,54 & 6,35 & 3,46 \\
Valor predictivo positivo & 12,41 & 4,42 & 2,90 \\
Valor predictivo negativo & 83,87 & 36,66 & 21,81 \\
Exactitud & 29,07 & 9,27 & 5,51 \\
\hline
\end{tabular}

TABLA 7. Tablas de contingencia $2 \times 2$ y su resolución a distintas probabilidades de predicción de mortalidad mediante MPM II 0

\begin{tabular}{|lccc|}
\hline Probabilidad & $20 \%$ & $50 \%$ & $70 \%$ \\
Sensibilidad & 81,66 & 61,66 & 45 \\
Especificidad & 29,71 & 10,61 & 5,42 \\
Valor predictivo positivo & 14,12 & 8,89 & 6,30 \\
Valor predictivo negativo & 91,97 & 66,17 & 4,75 \\
Exactitud & 36,15 & 16,94 & 10,33 \\
\hline
\end{tabular}


TABLA 8. Tablas de contingencia alrededor de la probabilidad de muerte establecida alrededor del valor de predicción medio (media aritmética)

\begin{tabular}{|l|c|c|c|}
\hline & EPEC & SAPS II & MPM II 0 \\
\hline & 10,46 & 19,35 & 24,54 \\
Sensibilidad & 16,94 & 71,69 & 81,66 \\
Especificidad & 33,96 & 22,54 & 23,82 \\
Valor predictivo positivo & 3,44 & 12,41 & 13,17 \\
Valor predictivo negativo & 74,61 & 83,87 & 90,17 \\
Exactitud & 31,88 & 29,07 & 30,99 \\
\hline
\end{tabular}

EPEC: estimación pronóstica en enfermos críticos.

probabilidad de muerte establecida como del valor de predicción medio (media aritmética).

Las ecuaciones de regresión lineal, que permiten la interutilización de los distintos métodos de estimación pronóstica de supervivencia, tienen unos excelentes coeficientes de correlación $(\mathrm{R})$ con una significación de $\mathrm{p}<0,05$. Los valores son: a) EPECSAPS II: R 0,658; p < 0,001; b) EPEC-MPM 0: R 0,704; p < 0,001 y c) SAPS II-MPM 0: R 0,732; p < 0,001 .

La calibración de los tres métodos (con las limitaciones a que obliga la restricción de los intervalos de probabilidad a menos de $50 \%$ para EPEC), mediante el test $\mathrm{C}$ de Hosmer-Lemeshow, puede calcularse a partir de las tablas 1, 2 y 3. Proporciona los siguientes valores: para EPEC, Chi cuadrado 9,66, p $<0,05$; para MPM 0, Chi cuadrado 23,76; $<<0,001$, y para SAPS 2 Chi cuadrado 12,43 ; $\mathrm{p}=\mathrm{NS}$, resultando pues que para este tamaño muestral sólo SAPS II está bien calibrado en su capacidad de predicción de mortalidad.

La capacidad discriminante de supervivencia se ha estudiado mediante el cálculo de las áreas bajo las curvas ROC (figs. $1 \mathrm{~A}, \mathrm{~B}$ y C) y proporciona los siguientes resultados: 0,836 (IC 95\%: IC 0,7810,891; $<<0,001)$ para EPEC, $0,839(0,780-0,899 ; \mathrm{p}$ $<0,001)$ para MPM II 0 y $0,827(0,777-0,876$; p < $0,001)$ para SAPS II.

Finalmente, la coincidencia de capacidad predictiva fue estudiada mediante el método de concordancia de Bland-Altman. El análisis de los gráficos representados en las figuras 2 A y B permite comprobar que, en ambos casos, el IC al $95 \%$ es demasiado amplio (más de 80 puntos) y que en el caso de análisis de concordancia entre EPEC y SAPS II, más de un $5 \%$ de los puntos de coincidencia de los pares de predicciones están fuera del IC admitido. Podemos, pues, afirmar, que no existe concordancia entre las predicciones de mortalidad de EPEC y los otros dos métodos.

\section{DISCUSIÓN}

Cuando el investigador interesado en la aplicación de la práctica de la teoría del pronóstico se enfrenta a un nuevo método de estimación pronóstica (en este caso de supervivencia de pacientes críticos atendidos en un Servicio de Medicina Intensiva),

\section{ANEXO 2}

Definiciones de discriminación y calibración aplicadas a la elaboración de un sistema pronóstico

Discriminación
Capacidad del modelo para diferenciar entre los pacientes que
sobrevivirán de los que fallecerán. Se puede expresar mediante
el análisis de las curvas ROC (Receiver Operating
Characteristics).
Calibración
Grado de correspondencia entre las probabilidades estimadas
de mortalidad y la mortalidad realmente observada en la
población estudiada. Se puede expresar mediante el análisis del
test de bondad de ajuste de Hosmer-Lemeshow (Goodness).

debe tener permanentemente presentes las condiciones que debe cumplir en teoría cualquier método de estimación pronóstica de supervivencia.

Entre ellas están: la relación lineal de la estimación $(\mathrm{n}-1<\mathrm{n}<\mathrm{n}+1)$, la simplicidad de las variables utilizadas, la reproducibilidad de la estimación y la validación en muestra distinta de la utilizada para el diseño del sistema ${ }^{3,4}$. A estas condiciones relacionadas con la estrategia de diseño cabe añadir condiciones estrictamente de elaboración y estructuración matemática: una alta calibración, una alta discriminación y la posibilidad de que sus «prestaciones» (capacidad operativa de establecer un pronóstico) sean comprobadas en distintos ámbitos ${ }^{5}$. El anexo 2 ofrece las definiciones de calibración y discriminación.

En lo que se refiere a EPEC cabe destacar que algunas de estas condiciones no se cumplen en su descripción original, tanto en los aspectos de diseño (validación en muestra independiente) como en los de elaboración matemática (comprobación de prestaciones en distintos ámbitos). De ello nace la necesidad y el interés de esta aportación, y más cuando la simplicidad de diseño ofrece condiciones que pudieran hacer al método atractivo para su utilización en cualquier UCI.

La sencillez de elaboración de un método de estimación pronóstica debe apoyarse en la utilización de variables que puedan estar disponibles en cualquier ámbito de posible aplicación, con independencia de tecnologías y sofisticación de procedimientos de obtención; en este aspecto radica el principal capital potencial del método estudiado aquí. EPEC, al igual que otros métodos de estimación pronóstica de supervivencia de enfermos críticos, parte de esta premisa incuestionable. Ninguno de los sistemas más utilizados en la práctica (APACHE ${ }^{6,7}, \mathrm{SAPS}^{8,9}$, y los derivados del método MPM $^{10,11}$ ) utilizan variables que estén alejadas de la disponibilidad más rutinaria. No por incluir la determinación de los niveles de hormona del crecimiento, o complejas elaboraciones matemáticas a partir de variables fisiológicas simples, se mejora la prestación de una estimación pronóstica.

Fiel a esta concepción EPEC no incorpora ninguna variable que pueda ser catalogada de sofisticada 

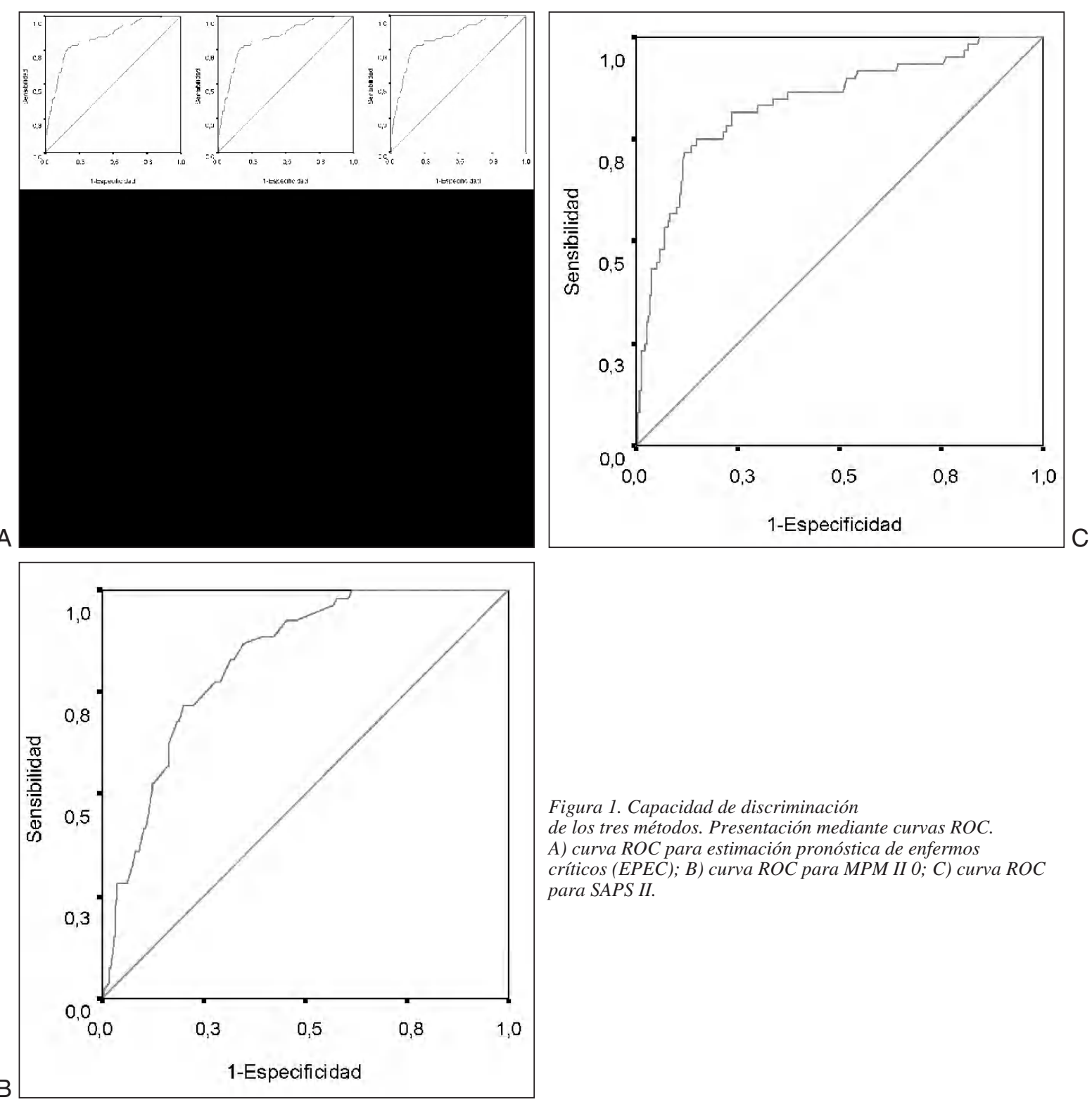

Figura 1. Capacidad de discriminación

de los tres métodos. Presentación mediante curvas ROC.

A) curva ROC para estimación pronóstica de enfermos

críticos (EPEC); B) curva ROC para MPM II O; C) curva ROC

para SAPS II.

o difícil de obtener, y éste es, junto con una simplificación importante de los procedimientos de cálculo necesarios para su aplicación (anexo 1), su principal atractivo.

Pero no es éste su único valor intrínseco. Desde los trabajos de Dragsted ${ }^{12}$ se acepta que el tiempo de retraso del ingreso del paciente en UCI en situación crítica es determinante para el resultado final. Este tiempo puede ser considerado de una forma bivalente: el retraso en identificar la situación de riesgo vital (al que podríamos llamar «retraso fisiopatológico», ya que está relacionado con las alteraciones que determina este retraso en la identificación del problema) y otra forma que sería la del retraso asociado a la imposibilidad de aceptar el ingreso del paciente candidato a tratamiento en UCI, ya sea por falta de disponibilidad de camas o por cualquier otro motivo. A éste podría llamársele «retraso logístico».

El trabajo original de Padrón et al, que describe EPEC$^{1}$, opta por esta segunda posibilidad, expresando este «retraso» en horas completas, y en nuestro conocimiento es el único sistema de estimación pronóstica de supervivencia que incorpora, como factor independiente, el retraso logístico, que en determinados centros puede tener un peso determinante.

De cualquier forma, y siguiendo este mismo hilo argumental, cabe pensar que de los dos retrasos considerados (el fisiopatológico y el logístico), es el primero el que se asocia a peores consecuencias y el que exige mayor esfuerzo para ser modificado, ya que el retraso que hemos llamado logístico depende, en última instancia, de un adecuado funcionamiento 

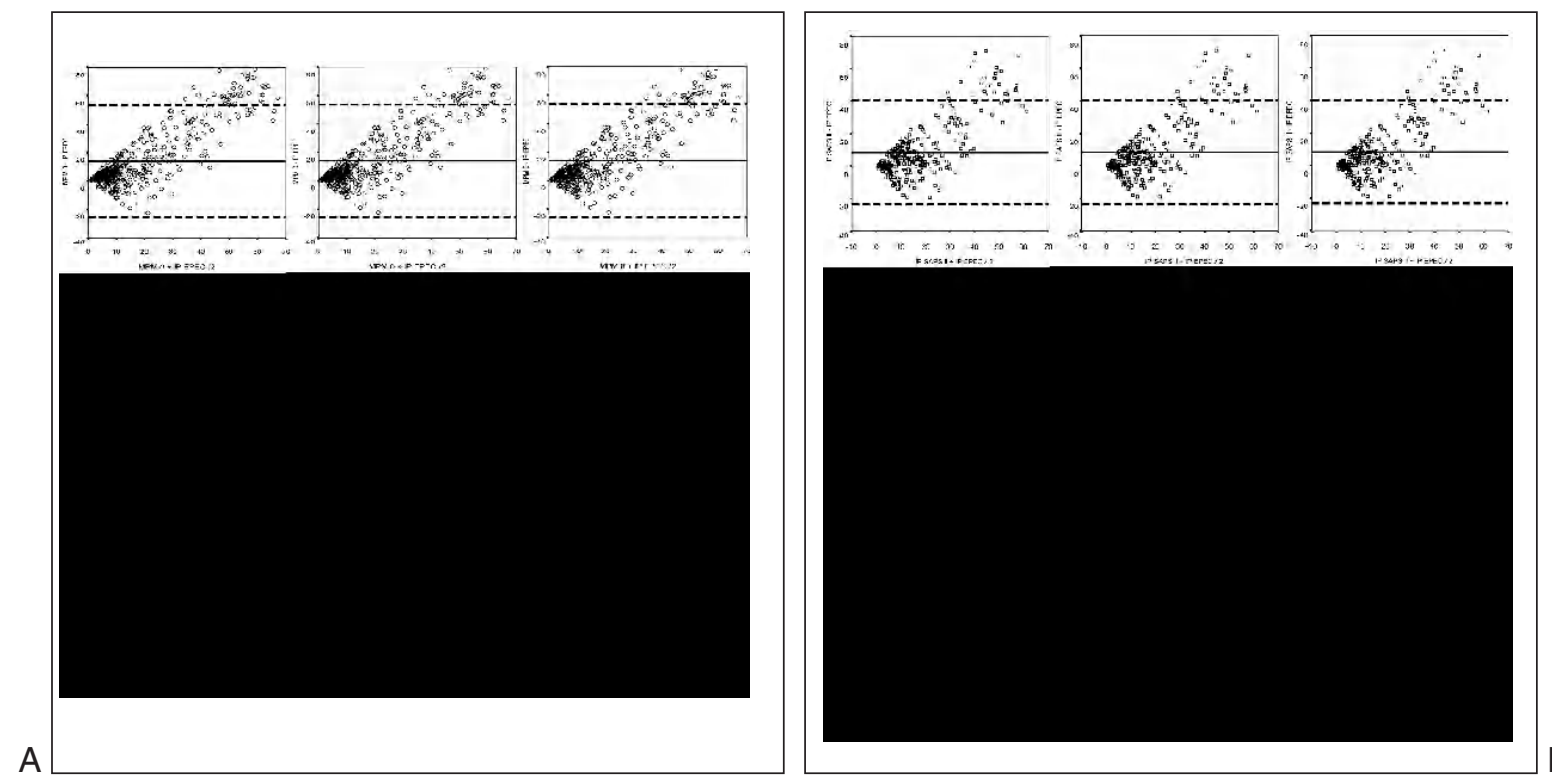

Figura 2. Análisis de concordancia predictiva mediante el test de Bland-Altman. A) Concordancia EPEC (estimación pronóstica en enfermos críticos)-MPM II 0; B) Concordancia EPEC-SAPS II.

de la propia UCI, mientras que el primero exige un cambio de mentalidad y de política de oferta y demanda por parte del conjunto de la estructura asistencial del centro.

Dentro de las promesas ofrecidas por los nuevos diseños de estimaciones pronósticas no se han conseguido solventar los déficit de prestaciones, ya denunciados recientemente por Knaus ${ }^{13}$, y que pueden resumirse en los dos aspectos clásicamente establecidos como limitaciones de todos los modelos de estimación pronóstica disponibles: uno hace referencia a la limitación de exactitud, que en la literatura se cifra en alrededor de un error por cada 10 casos predichos, es decir, en un 10\% de las predicciones. Esta limitación impide que la aplicación de los métodos predictivos de supervivencia pueda ser ascendida a niveles de criterio de decisión. Mientras exista una cierta incerteza ligada a la inexactitud (intrínseca) de predicción, las estimaciones pronósticas serán sólo instrumentos de trabajo en estudios de calidad ${ }^{4}$, pero no motivos de adopción de decisiones clínicas.

El otro consiste en la toma de decisiones asistenciales, ya que no se solventa el problema de las predicciones individuales frente a las de grupo. De hecho, ¿cómo puede interpretarse que un paciente tenga una probabilidad de sobrevivir (inversa del riesgo de muerte) de, digamos, 0,75 ? ¿Cuál es la traducción práctica para el paciente afecto de complicación perforativa secundaria a neoplasia intestinal, intervenido hace 12 horas y con un riesgo de muerte del 25\%? Esta es la principal objeción a los instrumentos predictivos, que su utilización en pacientes concretos e individuales es sólo orientativa y no permite ningún tipo de decisión concreta.

A la luz de la existencia, de la persistencia deberíamos decir, de estas limitaciones deben justificarse todos los intentos destinados a refinar la capacidad pronóstica, y en este sentido los esfuerzos de todos los investigadores deben ser vistos con respeto y merecer el máximo de atención, en función de su promesa implícita. Pero este aspecto estimulante de búsqueda del instrumento más adecuado no debe olvidar las condiciones establecidas para la creación de nuevas estrategias predictivas, y de ahí nuestro interés en validar (en una muestra distinta e independiente de la utilizada para el diseño) EPEC.

Desde el punto de vista de la validación de un nuevo método de estimación pronóstica, y al estudiar sus «prestaciones» en una población distinta de la inicialmente utilizada para el desarrollo de método, la cuestión fundamental es establecer si el nuevo método «funciona» mejor que los disponibles hasta ese momento, y comparar las predicciones obtenidas con el método incógnita frente a las recogidas con otros sistemas perfectamente consolidados. En nuestro estudio se ha comparado la capacidad predictiva de EPEC con las de MPM II 0 y SAPS II.

Desde el punto de vista de la discriminación entre supervivientes y no supervivientes es evidente que la capacidad de EPEC es claramente superponible a la de los otros métodos. El cálculo de las áreas bajo las curvas ROC para cada uno de los tres métodos proporciona valores (figs. $1 \mathrm{~A}, \mathrm{~B}$ y C) cuantitativamente tan próximos, que es evidente que no existen superioridades de un método con respecto a otro.

Estas aparentes similitudes de capacidad pronóstica se ven comprometidas al analizar la calibración obtenida y realizar los cálculos de resolución de las tablas de contingencia construidas alrededor de distintas probabilidades de mortalidad. EPEC no permite analizar adecuadamente la calibración al no existir, por este método, una probabilidad de muerte 
superior al 50\%, y a ello se debe añadir el análisis comparativo de la SMR frente a MPM II 0 y SAPS II. Mientras que estos métodos no ofrecen diferencias significativas entre ellos al establecer la comparación entre predicciones de mortalidad y mortalidad real, EPEC sí aporta diferencias significativas frente a los dos métodos, considerados aquí como gold standards.

En este mismo sentido debe interpretarse el resultado de los análisis de concordancia (test de BlandAltman) realizados, que demuestran que no existen coincidencias suficientes entre las predicciones de EPEC y las de SAPS II o MPM II 0.

A la luz de estos resultados creemos adecuado afirmar que, en su estado actual, EPEC no sólo no ofrece ventajas tangibles frente a otros métodos de estimación pronóstica más introducidos en la práctica (MPM II 0 y SAPS II), sino que su capacidad de pronosticar adecuadamente el riesgo de muerte es más que discutible.

Como resumen de nuestro trabajo se puede afirmar que éste es más un ejercicio encaminado a analizar la adecuación metodológica de un sistema de predicción de mortalidad, que a establecer su posibles bondades (que no las presenta) frente a sistemas más habituales en la práctica.

\section{AGRADECIMIENTOS Y CRÉDITOS}

Aspectos parciales de este trabajo han sido presentados en el XXXIX Congreso Nacional de la Sociedad Española de Medicina Intensiva, Crítica y Unidades Coronarias (SEMICYUC), celebrado en Tarragona en junio de 2004; y en el Congreso anual de la European Society of Intensive Care Medicine (ESICM), celebrado en Berlín en octubre de 2004.

\section{Declaración de conflicto de intereses}

Los autores han declarado no tener ningún conflicto de intereses

\section{BIBLIOGRAFÍA}

1. Padrón Sánchez A, Puga Torres M, Peña Dorado R, Bravo Pérez R, Quiñones Zamora A. Escala pronóstica del enfermo crítico (EPEC). Propuesta de una nueva escala predictiva. Primera versión. Rev Cub Med Int Emerg (ed. electrónica). 2002. Disponible en: http://bvs.sld.cu/revistas/mie/vol1_1_02/mie03102.pdf (consultado 20/8/04).

2. Padrón Sánchez A, Puga Torres M, Peña Dorado R, Bravo Pérez R, Quiñones Zamora A. Validación de la Escala Pronóstica del Enfermo Crítico I (EPEC I) comparada con las predicciones de mortalidad del APACHE II. Rev Cub Med Int Emerg (ed. electrónica). 2002. Disponible en: http://bvs.sld.cu/revistas/mie/ vol1_1_02/mie04102.pdf (consultado 20/8/04).

3. Abizanda R, Marsé P, Valle FX, Jordá R, López J. Consideraciones sobre la medida del nivel de gravedad en pacientes críticos. Su aplicación a un programa de calidad. Control de Calidad Asistencial. 1991;2:56-60.

4. Abizanda R, Rodríguez MT. The purpose and use of prognostic indices in critical care. Curr Opin Crit Care. 1996,2:214 20 .

5. Teres D, Lemeshow S. Using severity measures to describe high performance intensive care units. Crit Care Clin. 1993;9: 543-54.

6. Knaus WA, Zimmerman JE, Wagner DP, Draper EA, Lawrence DE. APACHE-acute physiology and chronic health evaluation: a physiologically based classification system. Crit Care Med. 1981;9:591-7.

7. Knaus WA, Draper EA, Wagner DP, Zimmerman JE. APA-

CHE II: a severity of disease classification system. Crit Care Med. 1985;13: 818-29.

8. Le Gall JR, Loirat P, Alperovitch A, Glaser P, Granthil C, Mathieu D, et al. A simplified acute physiology score for ICU patients. Crit Care Med. 1984;12:975-7.

9. Le Gall JR, Lemeshow S, Saulnier F. A new Simplified Acute Physiology Score (SAPS II) based on a European/North American multicenter study. JAMA. 1993;270:2957-63.

10. Lemeshow S, Teres D, Klar J, Avrunin JS, Gehlbach SH, Rapoport J. Mortality Probability Models (MPM II) based on an international cohort of intensive care unit patients. JAMA. 1993; 270:2478-86

11. Lemeshow S, Le Gall JR. Modeling the severity of illness of ICU patients. A systems update. JAMA. 1994;272:1049-55.

12. Dragsted L, Jorgensen J, Jensen NH, Bonsing E, Jacobsen E, Knaus WA, et al. Interhospital comparisons of patient outcome from intensive care: importance of lead-time bias. Crit Care Med. 1989; 17:418-22.

13. Knaus WA. APACHE 1978-2001: The development of a quality assurance system based on prognosis. Arch Surg. 2002; 137:37-41 